\title{
Process Quality Analysis of Products
}

\author{
K. S. Chen ${ }^{1}$, S. C. Chen ${ }^{2}$ and R. K. $\mathrm{Li}^{2}$ \\ ${ }^{1}$ Department of Industrial Engineering and Management, National Chin-Yi Institute of Technology, Taichung, Taiwan; ${ }^{2}$ Department of \\ Industrial Engineering and Management, National Chiao-Tung University, Hsinchu, Taiwan
}

Process capability indices are widely regarded as a flexible means of evaluating the quality and performance of a process. Accreditation by the Quality System Requirement QS9000 requires an agent to conduct a global evaluation on the process capability over the entire product family from a plant. Generally, the process capability of the whole product family is an important reference for evaluation when producing a new product or designing a new process. Therefore developing a scheme to evaluate the process capability of the whole product family or series is crucial for industry. This paper takes the production of capacitors as an example of a new method for evaluating the process capability of a whole product family. As the quality characteristic for producing capacitors is "the smaller the better", the upper specification limits of the capacitors of various specifications should first be established. The process capability index $C_{p u}$ for products for which "smaller is better" is then used as an evaluation tool to calculate the process capability indices for individual specified products. The relationship between the process capability indices and the yield will be studied. A process capability index is proposed to reflect the global yield of the whole product family and to serve as a reference for evaluating process capability for designing new processes or developing new products. The process capabilities for all products can thus be evaluated and the performance of each process can be evaluated according to the colour management method. Managers can determine easily whether products meet their specifications and take action to improve them if necessary, according to the colour management method.

Keywords: Colour management method; Process capability indices; Product family; Upper specification limit; Yield

\section{Introduction}

Process capability indices are currently regarded as effective in determining whether a process and/or a performance meets

Correspondence and offprint requests to: Dr K. S. Chen, National Chin-Yi Institute of Technology, No. 35, Lane 215, Sec. 1 Chung Shan Road, Taiping, Taichung, 411 Taiwan. E-mail: kschen@chinyi.ncit.edu.tw product requirements. Analysis can then lead to improvement of the process so that product failure can be prevented and the quality of products assured.

Many statisticians and quality control engineers have focused on process capability indices to establish more precise measures of process capability and performance in an increasing number of industries. Kane [1], Chan et al. [2], Choi \& Owen [3], Boyles [4,5], Singhai [6], Pearn et al. [7], and Chen [8] and others have all reported on this field. However, most studies were limited to discussing a process in terms of a single quality characteristic. According to Bothe [9], a product generally presents many important quality characteristics. A good product requires that all these characteristics meet specified requirements. Accreditation according to the Quality System Requirement QS9000 [10], requires that all agents conduct a global evaluation on the process capability indices over the whole product family produced in the plant. The requirement is also an important reference when developing a new product or designing a new process. The development of a method to assess the process capability of a whole product family by industry is thus important.

This paper discusses and schematises a method to evaluate the process capability for a whole family of products and capacitors are taken as an example. The quality characteristics for capacitors are all of the "smaller is better" type. Therefore, the unilateral specification limit indices $C_{p u}$ proposed by Kane [1] can be used to evaluate process capability. The relationship between the index $C_{p u}$ and process yield is a one-to-one process yield; therefore, from $C_{p u}$ we can deduce process yields. The relationship between the whole product family process yield and the process capability index $C_{T u}$ is studied in this paper.

Industries employ computer integrated manufacturing (CIM) in modern production, for example, for semiconductors and IC assemblies. This study considers the characteristics of capacitors obtained through sensors in a CIM environment. The average, $\mu$, standard deviation, $\sigma$, and indices, $C_{p u}$, can be calculated from measured data. The process capabilities for all products can thus be similarly evaluated and the performance of each process can be evaluated according to the colour management method (CMM) [10]. Managers can easily determine whether products meet specifications and take action to improve them if necessary. 


\section{Construction of Process Capability for Capacitors}

A company located in central Taiwan was selected for study. The company uses CIM to produce capacitors of various kinds according to CE directives and regulations. The main functions of capacitors are to store electric charge and provide other electric devices with current impulses of high voltage when needed. Examples include flashlights in cameras and control devices in computers. According to the CE directives and regulations, a capacitor's ability to maintain a current is a main quality characteristic. High current loss results in inefficient impulse output, which can affect the performance of other electric devices. For example, exposure failure can be expected if a flashlight cannot provide sufficient luminosity owing to its capacitors having a high rate of loss of charge. The decline of current from a capacitor is measured by the value of the loss corner. This value represents the loss of charge for a capacitor charged with a d.c. current for $5 \mathrm{~min}$ at a room temperature of $25^{\circ} \mathrm{C}$.

The quality characteristic of a capacitor, such as the loss corner is of "the smaller the better" type. Its upper specification limit is determined by some functions of the current and voltage capacity. Different current and voltage capacities result in different upper specification limits for the loss corners of capacitors. The company mentioned above produces capacitors of 12 different voltage capacities and 10 different current capacities yielding a total of 120 different specifications. Let $C$ and $V$ denote the current and voltage capacities, respectively. The upper specification limit for the loss corner of a capacitor whose voltage capacity $V$ is less than or equal to $100 \mathrm{SV}$ is

$$
U S L=(0.01) \times C \times V+I_{1} \times 3
$$

where $I_{1}=1$ when $(0.01 \times C \times V)<3$ and $I_{1}=0$, otherwise. In cases where $V>100 \mathrm{SV}$, the limit is given by

$$
U S L=(0.06) \times C \times V+I_{2} \times 10
$$

where $I_{2}=1$ when $(0.06 \times C \times V)<10$ and $I_{2}=0$, otherwise. The upper specification limits for the 120 different capacitors were calculated and given in Table 1 according to the above formulae.

For example, $I_{1}=1$ for a capacitor with $C=100 \mathrm{SV}$ and $V=1 \mu \mathrm{F}$ because $(0.01 \times C \times V)=1<3$. Therefore, $I_{1}=1$

$$
\begin{aligned}
U S L & =(0.01) \times C \times V+I_{1} \times 3 \\
& =(0.01) \times 100 \times 1+1 \times 3=1+3=4
\end{aligned}
$$

Another example is for a capacitor with $C=160 \mathrm{SV}$ and $V=220 \mu \mathrm{F}$. In this case,

$$
\begin{aligned}
I_{2} & =0 \text { for }(0.06) \times C \times V=(0.06) \times 160 \times 100 \\
=2112 & >10
\end{aligned}
$$

. Therefore,

$$
\begin{aligned}
U S L & =(0.06) \times C \times V+I_{2} \times 10 \\
& =(0.06) \times 160 \times 220+0 \times 10=2112
\end{aligned}
$$

Table 2 gives symbols used for different kinds of current and voltage capacities (example $V=A$, the current is $10 \mathrm{SV}, C=A$ the voltage is $1 \mu \mathrm{F} ; V=I$ the current is $100 \mathrm{SV}, C=P$ the voltage is $2.2 \mathrm{k \mu F}$.

The quality characteristic for corner losses is of "the smaller the better" type. Therefore the one-sided specification limit indices $C_{p u}$, proposed by Kane [1], can be used to evaluate process capability. The value of $C_{p u}$ is defined as follows.

$$
C_{p u \alpha \beta}=\frac{U S L_{\alpha \beta}-\mu_{\alpha \beta}}{3 \sigma_{\alpha \beta}} \quad\left(\alpha \in S_{C}, \quad \beta \in S_{V}\right)
$$

where $U S L_{\alpha \beta}, \mu_{\alpha \beta \times}$, and $\sigma_{\alpha \beta}$ are the average, the standard deviation, and upper specification limit of the loss corners, respectively. Then, the current $C=\alpha$ and voltage $V=\beta \times C_{p u \alpha \beta}$ represents the process capability index for a capacitor with a current capacity of $\alpha$ and a voltage capacity of $\beta$, where $\alpha \in S c=\{A, B, D, E, F, G, H, I, J, K$,$\} and \beta \in S v=\{A, B$, $D, E, F, G, H, I, J, K, L, M\}$

The capacitors produced by the company have 120 different specifications resulting from 12 different voltage and 10 different current capacities. Table 3 gives the process capability for

\begin{tabular}{|c|c|c|c|c|c|c|c|c|c|c|c|c|}
\hline$V$ & 10 & 16 & 25 & 35 & 50 & 63 & 80 & 100 & 160 & 250 & 350 & 450 \\
\hline 1 & 3.1 & 3.16 & 3.25 & 3.35 & 3.5 & 3.63 & 3.8 & 4 & 19.6 & 15 & 21 & 27 \\
\hline 3.3 & 3.33 & 3.528 & 3.825 & 4.155 & 4.65 & 5.079 & 5.64 & 30.3 & 31.68 & 49.5 & 69.33 & 89.1 \\
\hline 22 & 3.52 & 5.2 & 5.5 & 7.7 & 11 & 13.86 & 17.6 & 22 & 211.2 & 330 & 462 & 594 \\
\hline 47 & 4.7 & 7.52 & 11.75 & 16.45 & 23.5 & 29.61 & 37.6 & 47 & 451.2 & 705 & 987 & 1269 \\
\hline 220 & 22 & 35.2 & 55 & 77 & 110 & 138.6 & 176 & 220 & 2112 & 3300 & 4620 & 5940 \\
\hline 470 & 47 & 7.52 & 117.5 & 164.5 & 235 & 296.1 & 376 & 470 & 4512 & 7050 & 9870 & 12690 \\
\hline 1000 & 100 & 160 & 250 & 350 & 500 & 630 & 800 & 1000 & 9600 & 15000 & 21000 & 27000 \\
\hline 3300 & 330 & 528 & 825 & 1155 & 1650 & 2079 & 2640 & 3300 & 31680 & 49500 & 69300 & 89100 \\
\hline 6800 & 680 & 1088 & 1700 & 2380 & 3400 & 4284 & 5440 & 6800 & 65280 & 102000 & 142800 & 163200 \\
\hline 10000 & 1000 & 1600 & 2500 & 3500 & 5000 & 6300 & 8000 & 10000 & 96000 & 150000 & 210000 & 270000 \\
\hline
\end{tabular}
different specifications.

For example, $C_{A B}$ represents the process capability index for a capacitor with a current capacity of $0.47 \mu \mathrm{F}$ and a voltage capacity of $10 \mathrm{SV}$.

\section{3. $\quad C_{p u}$ and Process Yield}

The process yield is \%Yield $=F_{x}(U S L)$ according to the conditions of one-sided specification limits for a "smaller the

Table 1. Upper specification limits for loss corners of capacitors with different current and voltage capacities.

$C$, current; $V$, voltage 
Table 2. Symbols used for different current and voltage capacities.

\begin{tabular}{|c|c|c|c|c|c|c|c|c|c|c|c|c|}
\hline$S$ & $A$ & $B$ & $D$ & $E$ & $F$ & $G$ & $H$ & $I$ & $J$ & $K$ & $L$ & $M$ \\
\hline$C$ & 1.0 & 3.3 & 22 & 47 & 220 & 470 & $1 \mathrm{k}$ & $3.3 \mathrm{k}$ & 160 & $10 \mathrm{k}$ & 250 & 450 \\
\hline
\end{tabular}

$C$, current; $V$, voltage; $k=1000$

$S$, symbols used for different kinds of current and voltage capacities

Table 3. Symbols used for quality indices associated with different current and voltage capacities

\begin{tabular}{llllll}
\hline \multirow{2}{*}{$\begin{array}{l}\text { Current capacity } \\
(C)\end{array}$} & \multicolumn{5}{c}{ Voltage capacity $(V)$} \\
\hline$A$ & $\ldots$ & $\beta$ & $\ldots$ & $M$ \\
$\vdots$ & $C_{p u A A}$ & $\ldots$ & $C_{p u A \beta}$ & $\ldots$ & $C_{p u A M}$ \\
$\alpha$ & $\vdots$ & $\vdots$ & $\vdots$ & $\vdots$ & $\vdots$ \\
$\vdots$ & $C_{p u \alpha A}$ & $\ldots$ & $C_{p u \alpha \beta}$ & $\ldots$ & $C_{p u \alpha M}$ \\
$K$ & $\vdots$ & $\vdots$ & $\vdots$ & $\vdots$ & $\vdots$ \\
\hline
\end{tabular}

better" type quality characteristic. Under normal conditions, the index $C_{p u}$ exhibits a one-to-one relationship with the process yield \% Yield. Their relationship can be expressed by

$$
\begin{aligned}
& \text { \%Yield }=\Phi\left(3 C_{p u}\right) \\
& p=\sum_{\alpha \in S_{C}} \sum_{\beta \in S_{V}} W_{\alpha \beta} p_{\alpha \beta}
\end{aligned}
$$

Where $\Phi$ is the cumulative distribution function for a standard normal distribution. The index $C_{p u}$ directly reflects the process yield because the process yield increases as the index $C_{p u}$ increases. For example, for a process yield, \% Yield $=99.865 \%$ the index $C_{p u}=1.0$. Similarly, Table 4 gives the process yields associated with each product specification in Table 3. In Table $4, P_{\alpha \beta}$ represents the process yield $\left(\%\right.$ Yield $\left.=p_{\alpha \beta}\right)$ for a capacitor with a current capacity of $\alpha$ and a voltage capacity of $\beta$. When $p_{\alpha \beta}=\left(3 C_{p u \alpha \beta}\right), \alpha \in S_{C}$ and $\beta \in S_{V}$. Let $N_{\alpha \beta}$ represent the number of capacitors produced. Let $N=\Sigma_{\alpha \in s_{c}} \Sigma_{\beta \in s_{v}} N_{\alpha \beta}$ and $W_{\alpha \beta}=\left(N_{\alpha \beta}\right) / N$. If each process is assumed to be mutually independent, the process yield for a series of products with different specifications can be expressed by

$$
p=\sum_{\alpha \in S_{C}} \sum_{\beta \in S_{V}} W_{\alpha \beta} p_{\alpha \beta}
$$

A process capability index, reflecting the product yield of a whole product family can be defined as,

Table 4. Process yields associated with different product specifications.

\begin{tabular}{lccccc}
\hline $\begin{array}{l}\text { Current capacity } \\
(C)\end{array}$ & \multicolumn{5}{c}{ Voltage capacity $(V)$} \\
\hline$A$ & $\ldots$ & $\beta$ & $\cdots$ & $M$ \\
\hline$\vdots$ & $p_{A A}$ & $\ldots$ & $p_{A \beta}$ & $\ldots$ & $p_{A M}$ \\
$\alpha$ & $\vdots$ & $\vdots$ & $\vdots$ & & $\vdots$ \\
$\vdots$ & $p_{\alpha A}$ & $\cdots$ & $p_{\alpha \beta}$ & $\ldots$ & $p_{\alpha M}$ \\
$K$ & $\vdots$ & $\vdots$ & $\vdots$ & & $\vdots$ \\
\hline
\end{tabular}

$$
C_{T u}=\frac{1}{3} \Phi^{-1}\left\{\sum_{\alpha \in S_{C}} \sum_{\beta \in S_{V}} W_{\alpha \beta} \Phi\left(3 C_{p u \alpha \beta}\right)\right\} \quad\left(\alpha \in S_{C}\right)
$$

According to the above definitions, the process capability and the yield of the whole product family are related to each other by the following one-to-one relationship:

$$
p=\Phi\left(3 C_{T u}\right)
$$

Therefore, the index $C_{T}$ satisfactorily represents the process capability and the yield of the whole product family.

The process capability index reflecting the product yield of a whole product family $C_{T u}$, and the index reflecting the product yield of on individual product $C_{p u i j}$ have a one-to-one relationship. Table 5 shows that the indices $C_{p u i j}$ and $C_{T u}$ fully reflect the process yield, since that process yield increases as the indices $C_{p u i}$ and $C_{T u}$ increase. For example, the process yield is 0.84134 when $C_{p u i j}$ or $C_{T u}$ is $\frac{1}{3}$. Table 5 gives the process yields associated with the indices.

\section{Provision Development of the Process Capability for the Product Family of Capacitors}

As mentioned above, the yield of a product family of capacitors is defined as the multiplication of the individual yields associated with the weight of output. Therefore, when the yield of the whole product family is equal to $p_{0}$, we cannot ensure that the yields of the individual product also equal $p_{0}$. It may be higher or lower than $p_{0}$. Similarly, when the index of whole product family is equal to $c$, we cannot ensure that the indices of each individual product are also equal to $c$. In other words, the process capabilities of each individual product should be higher than that of whole product family if some level of quality is required for the whole product family.

Table 5. The process yield $p \%$ related to index $C_{I}$ from $\frac{1}{3}$ to 2.0 .

\begin{tabular}{ll}
\hline$C_{I}$ & Process yield $p \%$ \\
\hline$\frac{1}{3}$ & 0.841344746 \\
$\frac{2}{3}$ & 0.977249868 \\
1.0 & 0.998650102 \\
$4 / 3$ & 0.999968329 \\
$5 / 3$ & 0.999999713 \\
2.0 & 0.999999999 \\
\hline
\end{tabular}


Table 6. Process capability constant quality and light.

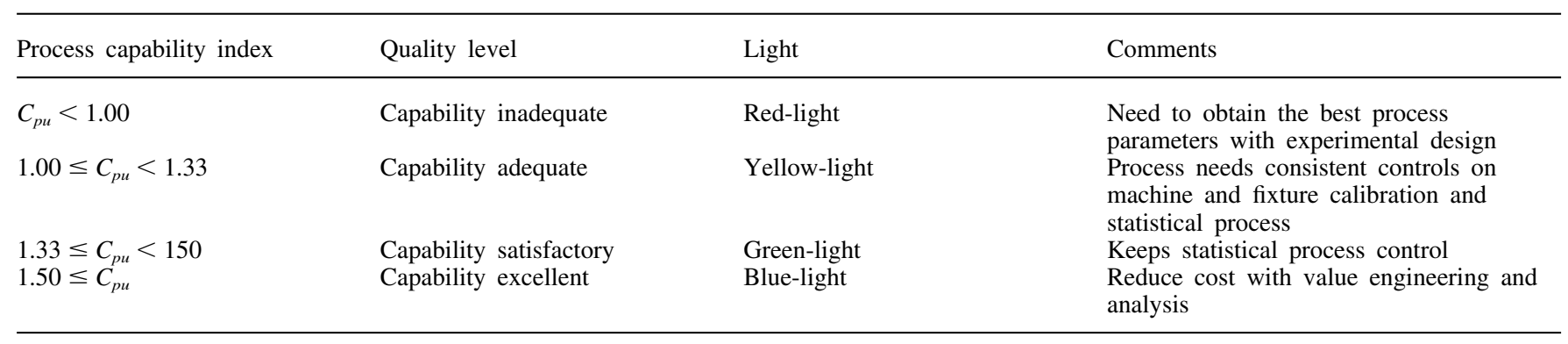

Table 7. Process capability and production weight.

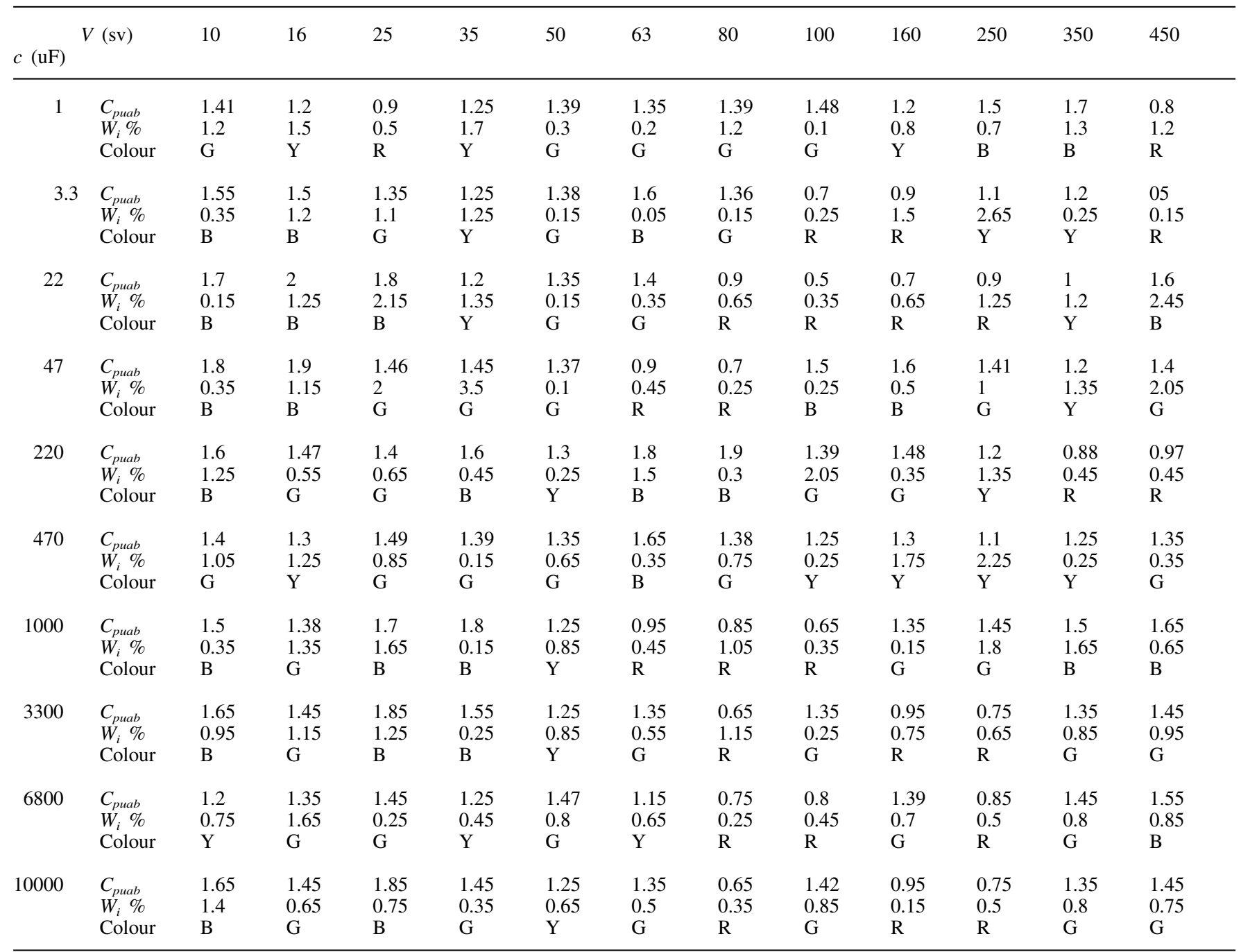

$C$, current; $V$, voltage; B, blue light; $\mathrm{G}$, green light; $\mathrm{Y}$, yellow light; $\mathrm{R}$, red light 
Then,

$$
\begin{aligned}
p & =\sum_{\alpha \in S_{C}} \sum_{\beta \in S_{V}} W_{\alpha \beta} p_{\alpha \beta} \\
& =p_{0} \times\left(\sum_{\alpha \in S_{C}} \sum_{\beta \in S_{V}} W_{\alpha \beta}\right)=p_{0} \\
C_{T u} & =\frac{1}{3} \Phi^{-1}\left\{\sum_{\alpha \in S_{C}} \sum_{\beta \in S_{V}} W_{\alpha \beta} \Phi\left(3 C_{p u \alpha \beta}\right)\right\} \\
& =\frac{1}{3} \Phi^{-1}\left\{\Phi(3 c) \times\left(\sum_{\alpha \in S_{C}} \sum_{\beta \in S_{V}} W_{\alpha \beta}\right)\right\}=c
\end{aligned}
$$

As mentioned above, from a practical perspective, we can only request that an individual process capability index be equal to $c$. We can then ensure that the whole product family process capability index will also be equal to $c$. The specifications of all products measured on the production line can be obtained at any time through sensors since all the capacitors are produced in a CIM environment. The mean, $\mu$, and standard deviation, $\sigma$, can be calculated from measured data according to the upper specification limits given in Table 1, and thus the value of $C_{p u}$ can be obtained. The process capabilities for all products can thus be evaluated. These capability indices $C_{p u}$ may be used to indicate the quality level of the product. The CMM [11] is a very human friendly management tool. We can assume that a value of $C_{p u}$ of less than 1 indicates that the process capability lies in a red-light area; between 1 and
1.33 , in a yellow-light area; between 1.33 and 1.50 , in a greenlight area; and above 1.5, in a blue-light area. Table 6 gives the process capability of an individual product corresponding to these four areas. Some further controls and amendments for each category are also represented in the table.

In Table 6, a red-light indicates that process capability is inadequate and so action is required to obtain the best process parameters by experimental design; a yellow-light indicates that the process is adequate and that the consistent control of machines, fixture calibration and statistical process control must be maintained; a green-light indicates that the process is satisfactory and statistical process control must be maintained; a blue-light indicates that the process is excellent and exceeds the requirements, so it may be possible to reduce costs through value engineering and analysis.

\section{Case Study}

The company produces capacitors of 120 different specifications with different voltage capacities and current capabilities. The loss corner data is collected by an automatic testing system. From this data, we can calculate the process mean and standard deviation and process capability. We can then transform the process capability of an individual product to the whole product family. Practically, we can only require that an individual process capability index and the whole product

Table 8. Exceptional light and production weight.

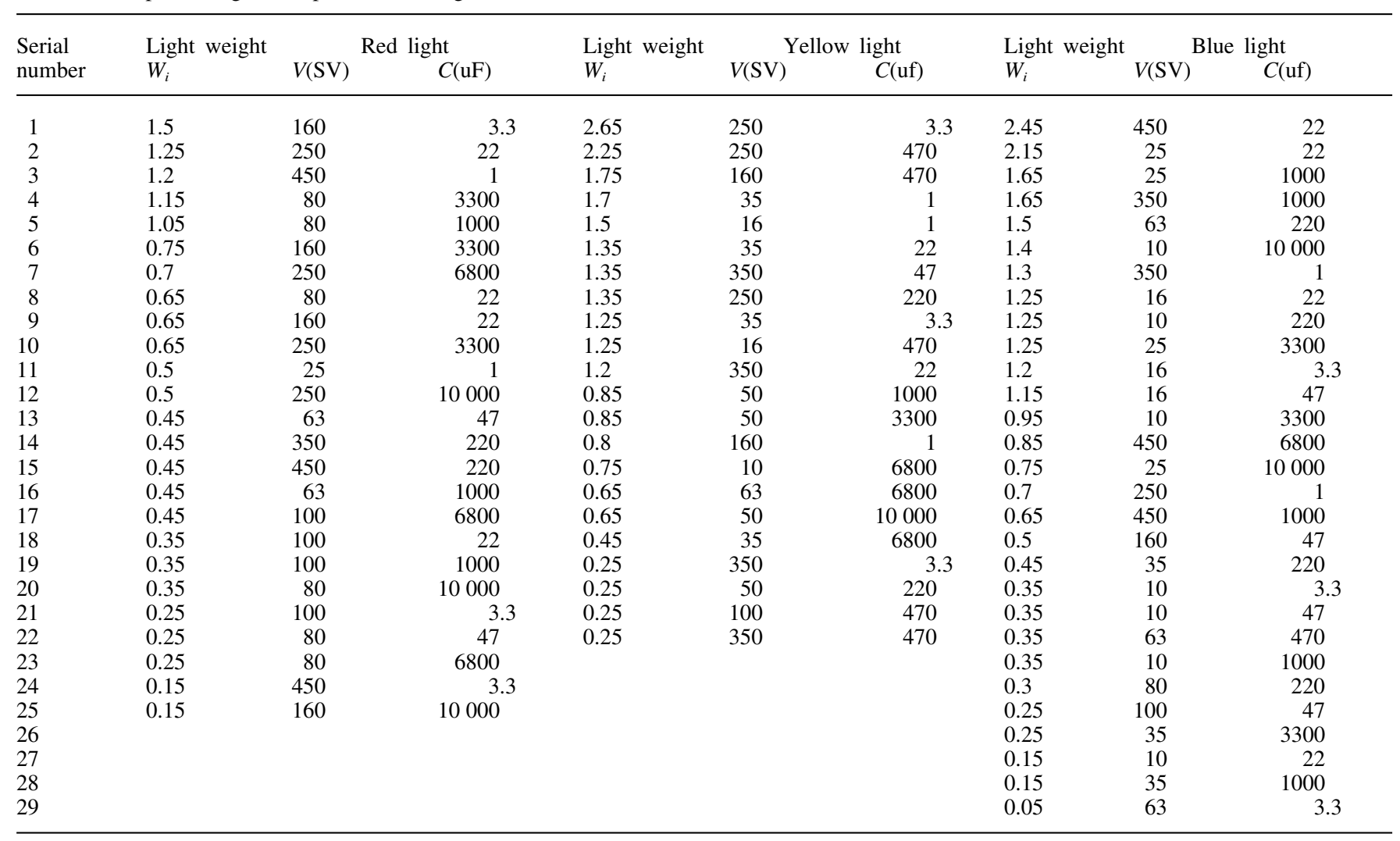

$C$, Current; $V$, Voltage 
family process capability index lie in the green-light area. We must then determine the priority of improvements to ensure optimal improvements in the shortest time period. Tables 6, 7 and 8 allow us to set such a priority.

According to Table 8, 25 products lie in the red-light area, 22 in the yellow-light area, and 29 in the blue-light area. Process capability in the red-light area is insufficient and so it is necessary to obtain the best process parameters using experimental design. The yellow light area indicates that the process capability is sufficient and consistent machine control of fixture calibration and statistical process control is required. The bluelight area indicates that process capability exceeds the requirements and so cost may be reduced with value engineering and analysis. We can refer to the colours and the yield weightings of Table 8 to determine the priority of product improvements.

\section{Conclusions}

Although they are increasingly popular, the application of process capability indices is mostly confined to the analysis of single quality characteristics and multiprocess performance. However, the safety code currently requires examination of the process capabilities of an individual, a series, and a family of products. Therefore, evaluating the process capability of a product family is very important for a plant governed by the safety code or QS9000.

We considered a family of capacitors with the same quality characteristics, but different specifications and production weightings. The process capability or process yield of capacitors with individual specifications was first calculated and then transformed to the capability for the whole family of products. A cross-reference table was also established to relate the process capabilities of a family to those of its individual products. Another table displayed different specifications and production weights for the CMM. A process capability management and improvement sequence can be established according to the colour and production weight. The process capabilities and yields of a family of products can be predicted since the process is monitored. Proper and prompt controls and changes can then be made to the process if necessary.

Results of this study can be extended to an examination of the process capabilities of a whole plant, producing products with different quality characteristics. Improvement of process capability with resource constraints can also be studied.

\section{References}

1. V. E. Kane, "Process capability indices", Journal of Quality Technology, 18(1), pp. 41-52, 1986.

2. L. K. Chan, S. W. Cheng and F. A. Spiring, "A new measure of process capability $C_{p m}$ ", Journal of Quality Technology, 20(3), pp. $162-175,1988$

3. B. C. Choi and D. B. Owen, "A study of a new capability index", Communications in Statistics: Theory and Methods, 19, pp. 12311245, 1990.

4. R. A. Boyles, "The Taguchi capability index", Journal of Quality Technology, 23(1), pp. 17-26, 1991

5. R. A. Boyles, "Process capability with asymmetric tolerances", Communications in Statistics: Computation and Simulation, 23(3), pp. 615-643, 1994.

6. S. C. Singhai, "Multi-process performance analysis chart (MPPAC) with capability zones", Quality of Engineering, 4(1), pp. 75-81, 1991.

7. W. L. Pearn, S. Kotz and N. L. Johnson, "Distributional and inferential properties of process capability indices", Journal of Quality Technology, 24(4), pp. 216-231, 1992.

8. K. S. Chen, "An incapability index with asymmetric tolerances", Communication Statistics Sinica, 8(1), pp. 253-262, 1997.

9. D. R: Bothe, "A capability study for an entire product", ASQC Quality Congress Transactions, pp. 172-178, 1992.

10. Quality System Requirement QS9000, 3rd edn, 1988.

11. Ming Guo Zhuang Color Management of Enterprise, 7th edn (in Chinese), 1992 